\title{
Impact of a multimodal anatomy CPD course on intravenous cannulation skills - An Irish radiographers and radiation therapists' perspective
}

\author{
Mutahira Lone $^{1}$, Ahmad H. Sheikh ${ }^{1}$, Andreea Factor ${ }^{1}$, Niamh Moore ${ }^{2}$, Muhammad A. Javaid ${ }^{3}$ \\ ${ }^{1}$ Department of Anatomy, University College Cork, Cork, Ireland \\ ${ }^{2}$ Department of Radiography, University College Cork, Cork, Ireland \\ ${ }^{3}$ Department of Anatomy, Anglia Ruskin University, Chelmsford, United Kingdom
}

\section{SUMMARY}

Radiographers and radiation therapists continually upgrade their knowledge to remain informed and competent in modern radiological imaging techniques. Despite the generally agreed upon significance of anatomy for successful interpretation of imaging modalities, its link with clinical performance of radiographers and radiation therapists has not been highlighted before.

The above-stated gap in the literature was addressed by employing an anatomy-based CPD course and investigating its influence on intravenous cannulation and administration skills of 10 radiographers and 4 radiation therapists. The course comprised interactive anatomy tutorials (employing vascular histology e-modules and vascular prosections), lectures and simulation sessions to practice IV cannulation. Likert questionnaires were employed to gauge attitudes and interests, prior to and after the course.

Corresponding author:

Muhammad Asim Javaid MD, PhD, Senior Lecturer. Department of Anatomy, School of Medicine, Anglia Ruskin University, Bishops Hall Lane, Chelmsford, United Kingdom. Phone: +353-21-4205494. E-mail: Muhammad.javaid@aru.ac.uk
At the end of the course, participants perceived anatomy prosections/dissection more useful compared to other learning modalities, including social media, textbooks, e-learning, plastic models and lectures. This corresponded with a significant increase in their perceived level of anatomy knowledge and confidence in identifying structures on prosections, and tracing vessels on radiographs and body surface. Anatomybased CPD course was valued with regards to their career progression and enhancement of anatomical knowledge. Furthermore, the use of prosections and hands-on practical sessions was highly commended and a desire to attend similar future courses was expressed. Lastly, participants identified factors facilitating or hindering course attendance and offered advice to improve efficacy of future courses.

We conclude that an interactive anatomy-based CPD-employing multimodal pedagogies-can be effective in enhancing anatomy knowledge and clinical competence of radiographers and radiation therapists.

Submitted: February 14, 2021. Accepted: November 17, 2021

https://doi.org/10.52083/QPAW2060 
Key words: Continuous professional development (CPD) - Radiographers - Radiation therapists - Anatomy - IV cannulation - Human prosections

\section{INTRODUCTION}

Anatomy has long been considered an essential part of medical education (McLachlan and Patten, 2006; Patel and Moxham, 2006; Drake et al., 2009). The significance of the link between a deep-rooted understanding of human anatomy and enhanced clinical interpretation and patientmanagement skills of healthcare professionals has been widely recognized (Turney, 2007; Arraez-Aybar et al., 2010; Martin et al., 2014). The practice of various allied healthcare professions, including radiography and radiation therapy, is heavily patient-centered. Hence, anatomy education-in integration with radiography and radiation therapy-has an important role to play in ensuring professional competence and provision of the highest standard of patient care in these fields (Heptonstall et al., 2016). Moreover, amidst increasing demands on healthcare professionals, there is a critical need for radiographers and radiation therapists to continually update their clinical and professional knowledge, in alignment with the requirements of their rapidly evolving profession, and to become increasingly competent in using and interpreting radiological imaging and modalities (Wareing et al., 2017).

A deeper understanding of anatomical concepts is important in interpreting various imaging modalities (Sugand et al., 2010). However, despite its established value in medical education, a decline in the anatomy knowledge of both students and health practitioners has been documented over the course of their academic and clinical years (Waterson and Stewart, 2005; Turney, 2007; Hall and Durward, 2009; Craft et al., 2017). Although anatomy is taught in sufficient detail during undergraduate courses, a need for radiographers and radiation therapists to revisit regional and cross-sectional anatomy and correlate it with their radiological image-interpretation skills, still exists (Hall and Durward, 2009).
Continuous Professional Development (CPD) is essential to maintain and improve the skills and knowledge necessary to provide the best of care for the patients (Henwood et al., 1998; Marshall et al. 2008; Wareing et al., 2017). A recent survey showed that the Irish Radiation Therapy departments are routinely employing intravenous contrast (IVC) during radiation therapy (RT) planning, thus necessitating the acquisition of this skill (Minogue et al., 2019). Furthermore, the Health and Social Care Professionals Council has introduced a regulatory body (CORU) for the registration of radiographers and radiation therapists since 2013. As a regulatory body, CORU has exercised its role to protect the public by promoting high standards of professional conduct, education, training and competence through statutory registration of health and social care professionals (CORU, 2019). While CPD courses can be organized in numerous ways, three main models have been highlighted in the literature (Wallace and May, 2016; Grehan et al., 2018). These include an 'input-based' CPD that involves a traditional but passive approach of attending workshops, conferences, symposia without any assessment of the learning, which has taken place (Wallace and May, 2016; Grehan et al., 2018). On the contrary, an 'output-based' CPD focuses on the learning outcomes and its impact on an individual's clinical practice, to maintain and improve professional competencies (Wallace and May, 2016; Grehan et al., 2018). 'Hybrid' variant of CPDs lies somewhere in the middle and exercises a combination of input and output-based approaches (Doughty and Hodgson, 2009; Grehan et al., 2018).

Research studies have investigated various CPD outcomes for radiographers and radiation therapists; including the motivational instigators and barriers linked with radiography CPD courses (Grehan et al., 2018), relevant aspects of CPD for radiographers (Marshall et al., 2008) and rationale affecting the radiographers and radiation therapists' participation in CPD courses (Walsh and Craig, 2016). While previous studies explored barriers affecting CPD courses, including IV cannulation (De Boo et al., 2020), the novelty of this study is that it investigates the association 
between an anatomy teaching and learning CPD course and the cannulation skills of the course participants. It is our understanding that a link between basic anatomical knowledge and clinical performance of radiographers and radiation therapists has not been highlighted before.

The aim of the study was to investigate, via a questionnaire, the impact of a novel teaching and learning anatomy CPD course for radiographers and radiation therapists. The course was highly interactive and stimulated the active involvement of the participants. Moreover, they were encouraged to reflect and were provided ample opportunity to apply and practice their skills. The hands-on design of the course helped participants to identify and address any gaps in their clinical practice. In this study, an effective hybrid CPD model was used by employing multimodal pedagogical tools including prosections and modern pedagogies (TEL) and its association with the personal and professional development of the participants was explored.

\section{MATERIALS AND METHODS}

A CPD course pertaining to intravenous cannulation and administration was designed for radiographers and radiation therapists at University College Cork (UCC), Ireland. 14 participants (10 radiographers, 4 radiation therapists) attended the course. The CPD course was advertised on the website of UCC's Centre for CPD. In addition, advertising emails were distributed to the Radiography Service Managers and Radiation Therapy Service Managers nationally.

\section{Design of the CPD course}

The CPD course spanned over one and half days and comprised interactive anatomy tutorials, followed by didactic lectures and practical simulated training sessions pertaining to IV cannulation and administration.

\section{Interactive anatomy tutorials}

Day 1 of the CPD course commenced with 2-hours of anatomy tutorials on the circulatory system. The tutorials were conducted using human prosections, supplemented by plastic models and computer-assisted learning software (anatomy and physiology revealed ${ }^{\circledR}$ ). The tutorials were hosted in the Anatomy FLAME laboratory at UCC and were facilitated by senior anatomy demonstrators. Participants rotated through four interactive thirty-minute tutorial stations.

At the first station, the demonstrators helped the participants to recognize the histological appearances of the different types of blood vessels; elastic and muscular arteries, veins and capillaries. The underlying structural variations were linked with the functional specifications of different types of vessels and the modulation of the flow of blood in them. An online e-learning resource entitled the 'Blue Histology' (http://bit. ly/neurohistology) was used for teaching with participants asking questions throughout.

The learning sessions over the next three stations were conducted using formalin-preserved prosections of the human body. All participants wore gloves and manually manipulated the structures under supervision, to identify and appreciate anatomical relationships in a threedimensional haptic learning environment. A general overview of the human circulatory system was provided on the second station with participants identifying the major blood vessels of the trunk and the heart, and their features. Next, in the third station, participants identified the course of the arteries and veins-superficial and deep, in the shoulder, arm and forearm. They identified common sites of venous access. Moreover, the importance of arterial anastomoses in the shoulder and the upper limb and the significance of the flow of venous blood from the superficial to the deep veins was discussed with the demonstrators. Lastly, on reaching the fourth station, the participants were given a detailed overview of the vasculature of the pelvic region and the lower limb. The participants identified the principal arteries and veins within the hip, thigh, leg, ankle and foot regions. The tutorial facilitators demonstrated the localization of femoral, popliteal, dorsalis pedis and posterior tibial pulses, and described the accessible venous sites for emergency 'venous cutdown' procedures. In addition, the functional significance of arterial 
anastomoses in the hip and knee, the role of the perforator venous connections between the superficial and deep veins and the function of the 'soleus muscle pump' for venous return to the heart were also mentioned.

\section{Didactic lectures}

Following the 2-hour anatomy tutorial, the remainder of day 1 of the CPD course comprised a series of didactic lectures delivered by radiographers, nurses and radiologists experienced in IV cannulation and administration of agents. During these educational sessions, participants were familiarized with the following theoretical underpinnings of intravenous cannulation:

1. Pharmacological and physiological principles underlying a) safe administration of intravenous preparations (radiopharmaceuticals and contrast agents) in diagnostic and therapeutic imaging, and b) recognition and management of adverse reactions or anaphylaxis associated with intravenous administration of contrast media;

2. Undertaking of patient safety and infection control precautions associated with intravenous cannulations;

3. Establishment and implementation of departmental protocols for the administration of intravenous injections and the role of the radiographer and radiation therapist in this context;

4. Safe usage of power pump injectors;

5. Medico legal issues associated with IV cannulation and administration of contrast agents.

\section{Practical training session for intravenous cannulation and administration}

Day 2 was aimed towards providing practical training to the radiographers and radiation therapists regarding administering intravenous cannulation and injecting contrast media and radiopharmaceuticals during imaging procedures. The teaching reflected the general principles of the IIRRT guidelines of best practice. The half-day long practical training took place in the Advanced Clinical Skills Department of the university.

At the start, the candidates attended a 1-hour simulation session on the safe preparationtechnique for undertaking intravenous cannulations. This session was provided by an expert nurse in IV cannulation and was supervised by radiographers with at least 10 years' experience in IV cannulation. It involved the participants simulating the preparation of the cannulation equipment using all the appropriate infection control measures. Following the simulation, participants carried out several supervised IV cannulations on artificial training arms. There was 1 supervisor to 3 participants in these sessions, which lasted for 2 hours. The candidates learned the pathways that should be undertaken while carrying out cannulations, using a variety of cannulae and butterfly needles.

\section{Questionnaire design}

Questionnaires (1 and 2) were compiled to inquire about the opinion of radiographers and radiation therapists regarding the significance of anatomy teaching and learning for enhancing their clinical performance and their opinion regarding what they preferred in future $\mathrm{CPD}$ courses (Table 1). The questionnaires were modified versions of those employed earlier by Marshall and colleagues (2008) and Grehan and colleagues (2018), imparting validity to their design. The questionnaire design was further informed by piloting the questions across a group of radiographers and radiation therapists during the previous year (2018). The feedback acquired from the pilot study informed the adequacy of the final questionnaires and research design. However, the pilot data was not incorporated into the main study.

Questionnaire 1 was administered prior to the commencement of the CPD course on day 1. It inquired about the participants' demographics, their work experience and their anatomy teaching and learning experience (Table 1; part A).

Questionnaire 2 was administered at the end of the practical IV cannulation course on day 2 and inquired about participants' anatomy 
teaching and learning experience, their opinion about both the anatomical component and the IV cannulation segment of the CPD course, specific areas where they would like to see further taught anatomy content employed and their satisfaction level at the end of the course (Table 1; part B).

\section{Ethical approval}

The study received approval from the Institutional Social Research Ethics Committee (Log 2019-118).

\section{Statistical analysis}

All statistical analysis was carried out using Excel $^{\circ}$ and the Statistical Package for Social Sciences, SPSS ${ }^{\circ}$, version 22 (IBM Corp., Armonk, NY). Descriptive statistics (mean, SD and percentages) were provided for explaining participants' demographics (Table 2), their prior experience with regards to using various imaging modalities, exposure to anatomy pedagogies during undergraduate years, factors facilitating or hindering course attendance, participants' perceived importance of anatomy- based CPD for career progression, preference for various learning modalities (prosections, e-learning, etc.) and topics preferred in future CPD courses.

Since the opinion of participants regarding various elements in the questionnaire (Table 1) was gauged using Likert scales (ordinal data), hence non-parametric tests, such as, Friedman's paired ranking and Wilcoxon's signed ranking tests were employed. Friedman's paired ranking test followed by post-hoc Wilcoxon ranking tests for paired comparisons were used in order to analyse potential differences in participants' opinion pertaining to their perceived usefulness of various anatomylearning modalities, and differences in their confidence in being able to identify anatomical structures using various pedagogical modalities (prosections, radiographs, images, unlabeled diagrams). The latter (Wilcoxon ranking) test was also employed to compare participants' change in confidence in identifying anatomical vessels on radiographs vs body surface marking at the end of the CPD course.

Table 1. Design of the questionnaires.

A. Questionnaire 1 (administered prior to the CPD course) ${ }^{\text {a }}$

A1. Participants' characteristics: (1)Gender (2)Age (3) Nationality (4)Radiographer/radiation therapist (5)Graduation (institute, year) (6) Additional qualification.

A2. Work experience: (1)Practicing County (2)Type of institute (3) Number of people in the department (4)Time since in practice (5)Area of specialization (6) Career break taken (7) Experience working with imaging modalities.

A3. Anatomy teaching/learning experience: (1) Teaching pedagogies employed during undergraduate training (2)Perceived level of anatomy knowledge (3)Current usage of anatomy learning tools (4)Perceived usefulness of anatomy learning modalities (5)Perceived confidence of identifying (a-anatomical structures on various pedagogical modalities, b-specific vessels on radiographs and on human body).

\section{B. Questionnaire 2 (administered after the CPD course) ${ }^{\mathrm{a}}$}

B1. Anatomy teaching/learning experience: (1)Perceived level of anatomy knowledge (2)Perceived usefulness of anatomy learning modalities (3) Perceived confidence of identifying (a-anatomical structures on various pedagogical modalities, b-specific vessels on radiographs and on human body).

B2. Questions about the anatomy CPD course: (1)Importance for career progression? (2)Value added to knowledge of anatomy? (3) Willingness to (a-attend additional CPD courses? b-spend time on anatomy CPD?) (4)Beneficial aspects of the course for learning anatomy? (5) Areas which need more focus in the future? (6) Preferred learning materials in anatomy CPD? (7) When attending the CPD what were (a-underlying reasons? b-barriers encountered?) (8) Where did they hear about the course, their expectations and how to improve in the future?

B3. Earlier involvement and attendance in IV cannulation course earlier (Yes/No)

B4. Three specific topics or imaging modalities where the participants would welcome anatomy study materials in future CPD courses.

B5. Participants' course satisfaction.

${ }^{a}$ Questionnaires employed to gauge the attitudes and interests of radiographers and radiation therapists regarding an anatomy CPD course for IV cannulation, $\mathrm{CPD}=$ continuous professional development. 
Table 2. Demographics.



$\mathrm{SD}=$ Standard deviation, EU = European union, ${ }^{\mathrm{a}}=2$ participants (from Zimbabwe and India).

\section{RESULTS}

\section{Participants' characteristics}

The participants included 10 radiographers ( 2 males, 8 females) and 4 radiation therapists (1 male, 3 females), with a mean age of $29 \pm 6.2$ years (males $=28 \pm 5.3$ years, females $=29 \pm 6.6$ years) (Table 2). These were mainly Irish nationals (12/14 participants), who had graduated from Ireland and the United Kingdom. At the time of the study, all the participants were working in various counties of Ireland, with the majority (64.3\%) working within university-hospital-based settings (Table 2). Most participants (85.7\%) had graduated recently - within the last 10 years, with an average work experience of $2.6 \pm 1.3$ years (Table 2). Only two participants had graduated in 2001 and 2006, and possessed 18 and 13 years of experience practicing as a radiation therapist and a radiographer, respectively. In addition, the results revealed that 5 participants had previously taken a career break - two practitioners had taken a break for 3 months each, the other two for 12 months each, while one undertook a break for a time duration of 18 months. The career break was not influenced by the gender distribution of the participants ( 2 males, 3 females). Out of all the participants, $34.7 \%$ had been involved in performing intravenous cannulation prior to the course, while $21.4 \%$ had attended a similar CPD course earlier (Table 1-B3).

\section{Work focus}

The majority of participants (4 out of 14) specialized in working in CT. Several had worked in a variety of imaging modalities, including MRI, PET and the Cardiac catheterization laboratory (Table 1-A2-point 5). When inquired about the imaging modalities (Table 1-A2-point 7, Fig. 1), $>70 \%$ of participants had worked with fluoroscopy (78.6\%), plain film radiographs (78.6\%), pediatric imaging (71.4\%) and CT scanning (71.4\%). A lesser percentage of radiographers and radiation therapists had experience of working with trauma imaging (64.3\%), angiography and interventional imaging (50\%), GI studies (42.9\%), contrast agents (42.9\%), radiotherapy (28.6\%), mammography (14.3\%), MRI (14.3\%), nuclear medicine (7.1\%), ultrasonography $(7.1 \%)$ and PET imaging $(7.1 \%)$ (Fig. 1). 


\section{Anatomy teaching and learning experience}

When inquired about how anatomy was taught to the participants during undergraduate years (Table 1-A3-point 1), the results demonstrate that $93 \%$ of participants (13 out of 14) had been taught anatomy using PowerPoint lectures, with $64.3 \%$ been taught using dissection and plastic models, while prosections had been employed to teach the anatomy in only $7.1 \%$ of cases (1 participant) (Fig. 2). The data is not all-exclusive, with some participants having been taught using a combination of pedagogies during undergraduate years.

The results revealed a significant difference between the perceived usefulness of various modalities for learning anatomy, both before the CPD course (Table 1-A3-point 4, Fig. 3A, Friedman test; $\chi 2(5)=30.787, P<0.001)$ and after the CPD course (Table 1-B1-point 2, Fig. 3B, Friedman test; $\chi 2(5)=28.633, P<0.001)$. Additional analysis showed that before the CPD course, the usefulness of newer modalities, such as social media was rated significantly lower compared to all other modalities (Wilcoxon-signed rank test;
$P<0.05$ for social media vs. plastic models, $P<$ 0.01 for social media vs. e-learning, prosections/ dissection, textbooks and lectures). None of these mean perceived usefulness values correlated significantly with the age of the participants (Pearson correlation; $P>0.05$ ). The perceived usefulness of social media continued to rank lower even after the CPD course (Wilcoxon-signed rank test; $P<0.01$ for social media vs. lectures and prosections/dissection, $P<0.05$ for social media vs. e-learning, plastic models and textbooks). However, the perceived usefulness of prosections/ dissection increased significantly compared to all other anatomy learning modalities (Wilcoxonsigned rank test; $P<0.01$ for prosection/ dissection vs. social media and textbooks, $P<0.05$ for prosection/dissection vs. e-learning, plastic models and lectures).

When inquired if practitioners were using any learning tools prior to the CPD course to enhance their knowledge of anatomy (Table 1-A3-point 3 ), only 3 participants mentioned employing e-learning and textbooks, while others did not report using any learning resource at all.

\section{Participants' exposure to imaging modalities}

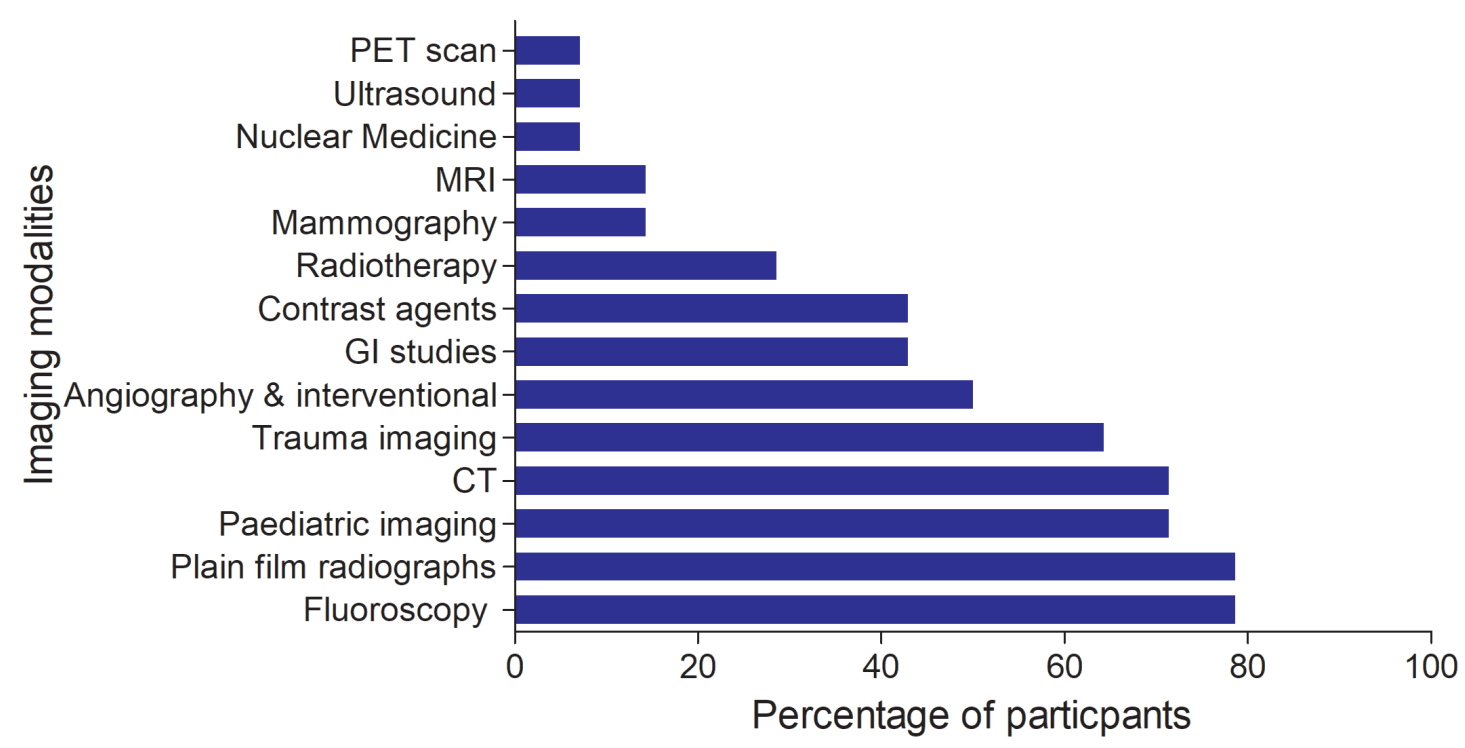

Fig. 1.- Percentage of participants (radiographers and radiation therapists) exposed to various imaging modalities. The greatest proportion of participants had worked with plain film radiographs and fluoroscopy, while the least proportion of participants were exposed to PET scan, ultrasound and nuclear medicine imaging modalities during their work experience. $\mathrm{CT}=$ contrast tomography, MRI=magnetic resonance imaging, $\mathrm{PET}=$ positron emission tomography, GI=gastrointestinal. 


\section{Tools for teaching anatomy during undergraduate years}



Percentage of particpants

Fig. 2.- How were you taught anatomy during undergraduate years? Majority participants had been taught anatomy using PowerPoint lectures, followed by plastic models, dissection, and prosections.

\section{Perceived usefulness of anatomy learning modalities}

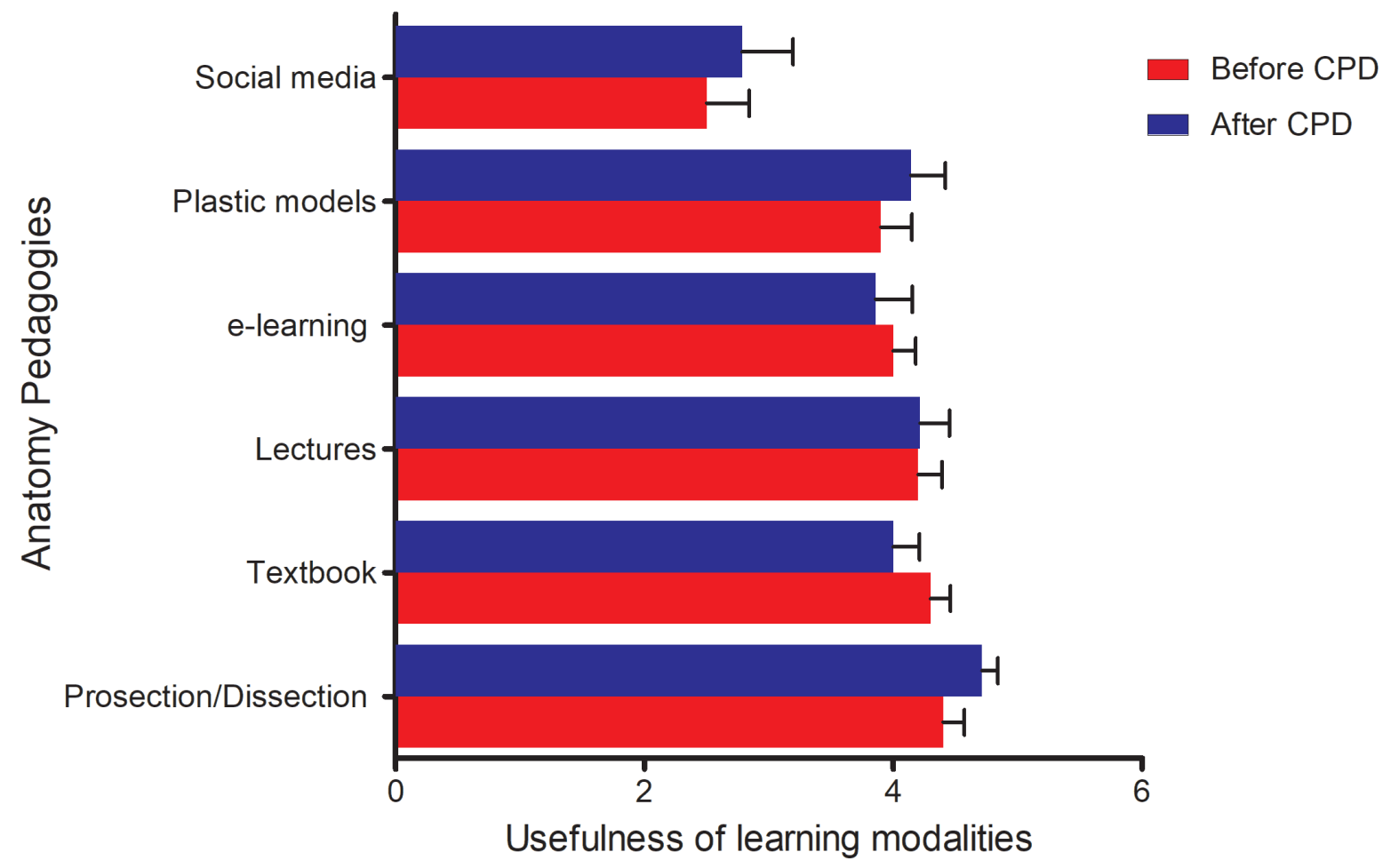

Fig. 3.- How would you rate the usefulness of following modalities for learning anatomy? (1=least useful, $5=$ most useful). The horizontal bars represent the participants' perceived usefulness of various modalities for learning anatomy, before (red bars) and after the CPD course (blue bars). The data demonstrates that social media was ranked significantly lower compared to all other modalities, before and after the CPD course. However, the perceived usefulness of the dissection/prosections increased significantly after the CPD course as compared to all other learning modalities $(\mathrm{P}<0.05)$. The error bars signify the standard error of means (SEM). 


\section{Perceived level of anatomy knowledge and confidence in identifying anatomical structures}

Prior to the commencement of the CPD course, the participants' average perceived level of anatomy knowledge was $3.3( \pm 0.27 \mathrm{SEM})$; on a scale of 1 to 5 (lowest to highest) (Table 1-A3point 2). While the work experience and the year of graduation of the participants were strongly correlated with each other $(r=0.969, P<0.01)$, neither of these two characteristic-features were significantly linked with the perceived level of anatomy knowledge (work experience; $r=0.317$, $P>0.05$, year of graduation; $r=0.226, P>0.05$ ). At the end of the CPD course, the participants seemed more confident regarding their level of anatomy knowledge (4.2 \pm 0.19 SEM), with the paired sample t-test results eliciting a significant increase in the mean perceived level of anatomy knowledge $(\mathrm{T}(13)=-2.738, P<0.05)$ (Table 1-B1point 1).

Prior to the CPD course, the participants showed a significant difference in their confidence in being able to identify structures between various pedagogical modalities (Table 1-A3-point 5a, Fig. 4A, Friedman test; $\chi 2(4)=33.809, P<$ 0.001). The confidence level was highest for the radiographs (Wilcoxon's signed rank test; $P<0.05$ for comparisons of radiographs vs. prosections, plastic models, unlabelled diagrams and images), and lowest for the prosections (Wilcoxon's signed rank test; $P<0.05$ for comparisons of prosections vs. images, unlabelled diagrams and prosections). The confidence levels for prosections (mean 2.8 $\pm 0.9 \mathrm{SEM}$ ) were not related to their earlier use of prosections $(r=-0.186, P>0.05)$ or dissection pedagogies $(r=0.069, P>0.05)$ for learning anatomy during the undergraduate years.

Following the CPD course, the participants were equally confident in identifying anatomical structures on all pedagogical modalities (Table 1-B1-point 3a, Fig. 4B, Friedman test; $\chi 2(4)=8.254$, $P>0.05)$. When compared with their confidence with the pedagogies prior to the CPD course, the results revealed that participants' confidence in identifying anatomical structures significantly increased across several pedagogies - including prosections $(P<0.01)$, plastic models $(P<0.05)$ and

\section{Confidence level in identifying anatomical structures}

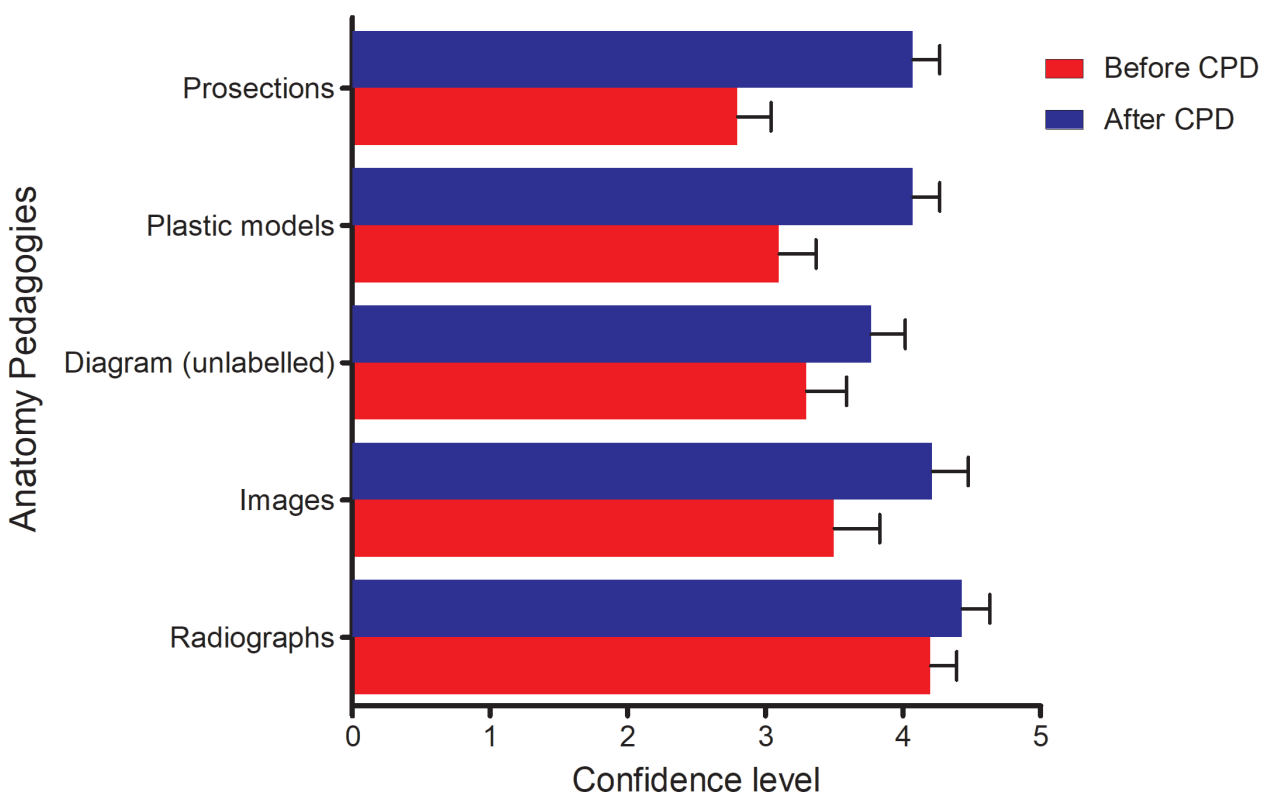

Fig. 4.- How would you rate your confidence at identifying anatomical structures in the following modalities/tools? (1=least confident, $5=$ highly confident). The horizontal bars represent the mean perceived level of confidence of the participants in identifying anatomical structures on various pedagogical modalities, before (red bars) and after the end of the course (blue bars). The error bars represent the SEM (standard error of mean). Results signify that participants' confidence in identifying anatomical structures increased at the end of the course, across all anatomical pedagogies. 
images $(P<0.05)$, but not for radiographs $(P>0.05)$ and unlabelled diagrams $(P>0.05)$ (Fig. 4A, B).

\section{Identification of specific anatomical vessels on the radiographs and on the human body}

The results did not reveal any significant difference in the confidence level of participants when identifying anatomical vessels on the radiographs as compared to surface-marking them on the human body (Fig. 5, Wilcoxon's signed rank tests for all vessels $P>0.05$ ).

The confidence level increased significantly by the end of the CPD course for most vessels, both in terms of identifying them on the radiographs (Fig. $5 \mathrm{~A}$ ) and surface-marking them on the human body (Fig. 5B). However, when compared against each other (post CPD, radiographs Vs. surface marking), the rise in confidence level was higher for surface marking for four vessels, namely cephalic, median cubital, popliteal and femoral veins, when compared to identifying them on the radiographs (Wilcoxon's signed rank test, $P<$ 0.05).

\section{Importance of the anatomy CPD course}

The radiographers and radiation therapists were asked about the importance of an anatomybased CPD for their career (Table 1-B2-point 1), $57.1 \%$ were of the view that an anatomy- based CPD is very important for their career and $42.9 \%$ perceived it to be important, while none considered it to be unimportant for their career progression. All participants reported that the course added to their knowledge of anatomy, with 4 participants believing that it provided an opportunity for them to review anatomy (Table 1-B2-point 2). In addition, everyone expressed their desire to attend additional similar CPD courses (Table 1-B2-point 3a), with 50\% willing to spend 1-5 hours, $35.7 \%$ willing to spend $5-10$ hours and remaining willing to dedicate $>10$ hours each year to attending similar CPD courses in the future (Table 1-B2-point 3b).

The participants had an equal preference for prosections/cadaver-based material (12 participants) and e-learning (11 participants) within an anatomy CPD course (Table 1-B2point 6). When inquired specifically about the beneficial aspects of the course (Table 1-B2-point 4), 7 out of 14 participants highly favoured the prosection-based anatomy teaching sessions and the practical sessions as part of the CPD course.

For the future CPD courses, a heavier focus was recommended on cross-sectional views of anatomy by 11 participants, vasculature by 7 participants and gross anatomy by 6 participants (Table 1-B2-point 5). When asked to pick three topics in which they would welcome anatomy

\section{A Confidence at identifying structures on radiographs}
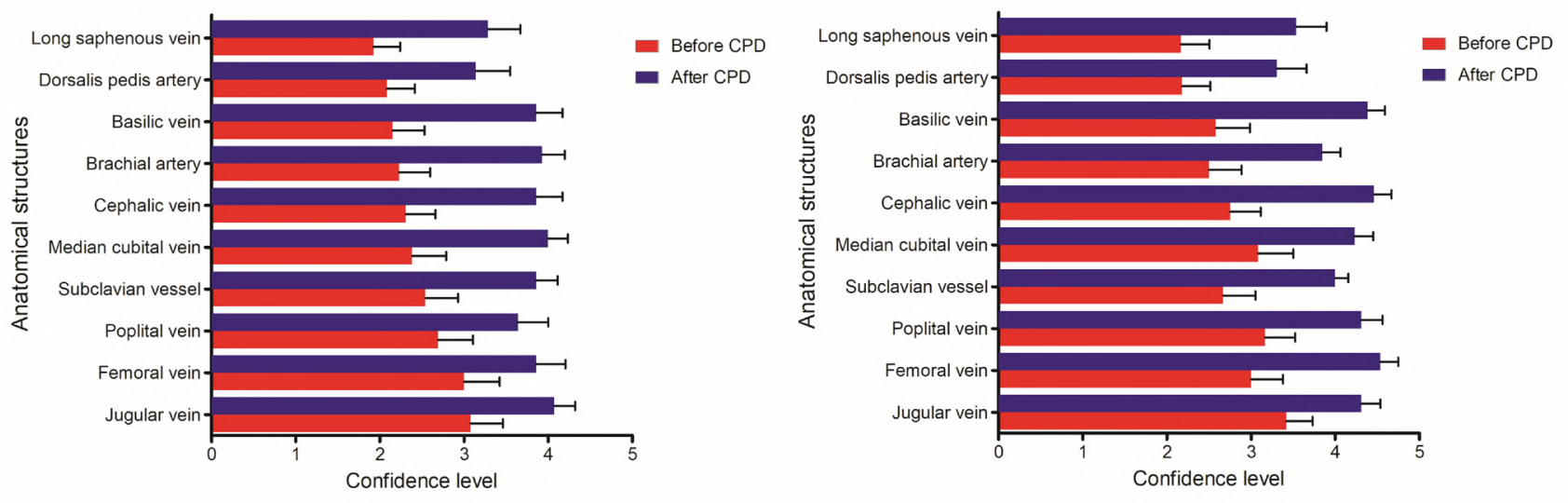

Fig. 5.- How would you rate your confidence at identifying the specific anatomical structures on radiographs and on patients (i.e., surface marking)? ( $1=$ least confident, 5 =highly confident). The horizontal bars represent the mean perceived level of confidence of the participants in identifying various arteries and veins (enlisted along the y-axis), before and after the CPD course - on the radiographs (A) and on the human body (B). The error bars signify the SEM (standard error of mean). Results reveal that participants' confidence in identifying anatomical vessels increased significantly for most vessels at the end of the CPD course, both on the radiographs and upon surface marking them on the human body. Note: ${ }^{*}=\mathrm{P}<0.05,{ }^{* *}=\mathrm{P}<0.01$. 
study materials during future CPD courses, CT was selected by 13 (out of 14) participants, MRI by 5 participants and cross-sectional anatomy and radiotherapy by 4 participants (Table 1-B5).

When inquiredabout the reasons for undertaking the CPD course (Table 1-B2-point 7a), developing new knowledge (11/14 participants), fulfilling the mandatory requirement (8/14 participants) and skill enhancement (12/14 participants) were considered equally important factors by the participants for attending the course. Funding, time allocation, location and staffing issues, management and employer attitude or support were highlighted as potential barriers for attending the CPD course (Table 1-B2-point 7b). Most participants heard about the course from their employer or at their workplace and attended it with the expectations of learning the skill of intravenous cannulation. Only 1 discovered the course online, while 1 participant attended the course with the expectation of acquiring the complete knowledge of vasculature of the upper and lower limbs (Table 1-B2-point 8).

\section{DISCUSSION}

Both radiographers and radiation therapists are highly motivated professionals who aim to provide the highest and safest standards of care for the patients. Therefore, it is important for these professionals to continue enhancing their knowledge by participating in CPDs relevant to their field of practice. This study is novel, as it provides an Irish perspective of radiographers and radiation therapists on the significance of anatomy teaching and learning during a CPD course on IV cannulation and administration. The participants came from various locations across the country, and had been working in different parts of Ireland, providing a homogenous perspective of Irish radiographers and radiation therapists, with opinion not being biased by any specific region. The participants attended an on-site IV cannulation CPD course for one and a half days, and were encouraged to actively engage and enhance their learning process using various interactive teaching approaches.
An overwhelming proportion of participants were from university-based hospitals, which could potentially reflect the greater inclination of faculty involved in teaching and training towards fulfilling their CPD requirements. A previous study by Marshall and colleagues (2008) also showed a higher participation of universitybased participants in CPD courses. Although their sample size was larger and encompassed 12 European countries, they did not include any Irish participants (Marshall et al., 2008). Hence, our study is novel, as it provides an Irish perspective on the significance of anatomy learning in an IV cannulation course.

An anatomy-based CPD was considered important by most Irish radiographers and radiation therapists for their career progression, in the context of enhancing their clinical IV cannulation skills. The key instigators were their interest in acquiring new knowledge and developing or enhancing their cannulation skills. All the participants agreed that the course added to their knowledge of anatomy, and agreed to attending similar courses in the future. Similar to other studies, funding, time allocation, location and staffing issues, management, and employed support were highlighted as potential barriers (Grehan et al., 2018).

The advent and rapid progression of the various cross-sectional medical imaging modalities (Marshall et al., 2008; Gore, 2020) were also reflected in our study by the fact that most participants' preferences for future CPD was an increased focus on crosssectional views of anatomy. The continuous improvement of knowledge in cross-sectional imaging is important for radiographers and radiation therapists (Marshall et al., 2008; Hardy and Snaith, 2009). Moreover, a heavier focus was recommended by participants on cross-sectional views of anatomy and greater incorporation of CT images in the future, similar to results reported by Marshall and colleagues. The results suggest that there is an appetite amongst the majority to undertake CPD, and therefore resources should be redirected to address these limiting concerns. Participants' suggestions should be incorporated into future 
courses for further enhancing the course quality standards and better meeting the radiographer's professional requirements.

Previously, Marshall and colleagues (2008) provided a general perspective of the Irish radiographers about the importance of $\mathrm{CPD}$. However, the significance of anatomy learning in the specific context of the development or the enhancement of their IV cannulation skill had not been explored. One must remain mindful of the fact that most of the study participants were at an early stage of their career, with only approximately one third having prior cannulation experience, or having previously attended a similar CPD cannulation course. Moreover, the majority of participants were working in university-based settings, with a potential greater interaction with the university academic staff members. This lack of experience and interaction with the academic staff members might have inclined them to having a positive opinion about the CPD course, and possibly added bias to the results. The latter is also supported by the fact that majority of study participants heard about the course at their workplace. Hence, while the study provided a novel Irish perspective, a larger-scale study with increasing number of participants across the country needs to be conducted to validate the results further.

Survey results from Grehan and colleagues (2018) showed that most radiographers believed that their CPD was self-directed, with most departments having yet to set up structured internal CPD programs. Our CPD course was sequentially well-structured in a manner where participants were taught the basic anatomy of the human vasculature, followed by didactic lectures, and then providing opportunity to the participants to practice their IV cannulation skills. Apart from the lack of structure, previous studies have also shown that formal didactic approaches which had been commonly employed for CPD learning, might not necessarily suit people with varying learning styles (Waterston and Stewart, 2005; Grehan et al., 2018). Some people learn better through hands-on experience and/or visual interaction with the study material or even observation of practice (Grehan et al., 2018). Previously, it was reported that traditional CPDs have little impact in enhancing professional practice (Davis et al., 1999; Bloom, 2005; Wallace and May, 2016). The current study offers valuable results to steer the discussion on this viewpoint by supporting the validity of the hands-on experience and clinical work, while the significance of formal didactic lectures is not undermined. The instructional design of our CPD incorporated hands-on teaching and learning of anatomy, using prosections where participants acquired a haptic feedback as they reviewed their anatomy knowledge of human vasculature. Moreover, a simulation course for practicing their IV cannulation skills was provided using artificial training arms, which participants could hold in their hands to acquire haptic feedback. The results suggest that it enhanced their knowledge of vascular anatomy, and participants had a positive opinion of the course and were interested in attending similar courses and considered it important for their career progression (Shanahan, 2016).

Currently, there is a gap in the literature on the use of social media within postgraduate radiography education (Sterling et al., 2017) with a lack of evidence on the benefits of radiographers using social media as a constructive, positive tool for ongoing CPD. Grehan et al. (2018) showed that although most radiographers were comfortable with using social media in general, more than $25 \%$ of study participants were unwilling to use social media as a mechanism of obtaining CPD, especially the older age group being more reluctant to use social media for CPD purposes (Grehan et al., 2018). Our results further reinforce these findings by showing a lack of perceived usefulness of social media for learning vascular anatomy for enhancing their IV cannulation skills.

Previously, in a large-scale study conducted across 12 European countries, Marshall and colleagues (2008) showed that most radiographers (67\%) prefer a combination of paper-based and e-learning methodologies as part of the pedagogical construct of the CPD courses, suggesting that they are receptive to creative ways for CPD delivery. However, the 
data on the provision of human cadaver material for CPD for radiographers was missing in the literature. The current study employed the use of human prosections to help radiographers and radiation therapists to learn the $3 \mathrm{D}$ anatomical relationships of vascular anatomy. And our results show that the participants' appreciation of the usefulness of prosections was significantly higher than that of social media and the former increased significantly after the course, compared to all other pedagogical tools, including lectures, videos, animations and models, etc. While these results highlight the educational efficacy of employing prosections (never done before) for radiography $\mathrm{CPD}$, it also highlights the usefulness of multimodal pedagogies employed in a structured fashion to enhance the knowledge and practical IV cannulation skills of the radiographers and radiation therapists. The results are useful in order to inform the instructional and experimental design of the future CPD courses for allied health professionals.

Our initial feedback suggests that the hybrid model of CPD is an excellent way to enhance the anatomy knowledge of radiographers and radiation therapists relevant to their field of practice.We consider that our CPD model is important, as the participants can integrate the anatomy knowledge achieved in their clinical practice. Ultimately, supporting and encouraging the radiographers and radiation therapists to engage and improve their continuous professional development is key in order to provide the best of care for the patients.

\section{Limitations of the study}

\section{Small sample size}

The results from the current study provide a novel Irish perspective on importance of anatomy learning and IV cannulation CPD. The sample size is limited; however, the results still hold paramount importance as they provide an Irish perspective, which is previously missing in the literature. We intend to continue the study for several years to increase our sample size and to follow the opinion of radiographers and radiation therapists in a prospective fashion.

\section{Triangulation of data-acquisition methods}

Data acquisition should be better triangulated by incorporating interviews and open-ended questionnaires, coupled with a qualitative analysis of the results acquired.

\section{CONCLUSION}

We conclude that the participants perceived anatomy prosections/dissection useful compared to other learning modalities. This corresponded with a significant increase in their perceived level of anatomy knowledge and confidence in identifying structures on prosections, and vessels on radiographs and body surface. The use of prosections and hands-on practical sessions was highly commended and a desire to attend similar future courses was expressed.

We conclude that an interactive anatomy-based CPD-employing multimodal pedagogies-is effective in enhancing anatomy knowledge, and can effectively contribute towards enhancing clinical competence of radiographers and radiation therapists.

\section{NOTES ON CONTRIBUTORS}

Dr Mutahira Lone, BDS. PhD is a lecturer and program coordinator for the MSc in Human Anatomy in the Department of Anatomy and Neuroscience at University College Cork. She also teaches gross anatomy to the MSc students and oral anatomy and histology to the dental students. Her research interest is focused on innovative and research-based pedagogies for anatomy students.

Ahmad H. Sheikh, MBBS, MRCS, MD is a Senior Medical Demonstrator in the Department of Anatomy and Neuroscience at the University College Cork, Ireland. He teaches Gross Anatomy and Neuroanatomy to a wide cohort of medical and health sciences' students using cadaver dissection, prosection, and a variety of modern anatomical teaching modalities. He devised a novel cadaver-based surgical skills and procedures workshop for medical students, as a new signature pedagogy in anatomical education.

Andreea Factor, M.D., MSc is a College Lecturer in the Department of Anatomy and Neuroscience, University College Cork, Ireland. She teaches in a number of programs in Medicine and Health as well as Biomedical Engineering. Her research interests include anatomy education, neurology and cardiology.

Niamh Moore, MSc is a diagnostic radiographer who holds an MSc in Computed Tomography. Niamh worked as a clinical radiographer for many years in advance of working in her current role as a lecturer on the MSc Diagnostic Radiography program in UCC. Niamh led in the development and running of the IV cannulation and administration course for Radiographers and Radiation Therapists in UCC.

Muhammad Asim Javaid, M.D., Ph.D is a senior lecturer in the Department of Anatomy at Anglia Ruskin University, 
Chelmsford, United Kingdom. He is the Year 2 academic lead for the MBCHB program in Anglia Ruskin. He teaches anatomy to medical and health science students using cadaverdissection, prosections and a variety of modern technological pedagogies. His research interests are focused on technologyenhanced learning in anatomy education.

\section{REFERENCES}

ARRAEZ-AYBAR LA, SANCHEZ-MONTESINOS I, MIRAPEIX RM, MOMPEO-CORREDERA B, SANUDO-TEJERO JR (2010) Relevance of human anatomy in daily clinical practice. Ann Anat, 192(6): 341-348.

BLOOM BS (2005) Effects of continuing medical education in improving physician clinical care and patient health: a review of systematic reviews. Int J Technol Assess Health Care, 21: 380-385.

CORU (2019) Guidance on Continuing Professional Development. Available from: https://coru.ie/files-education/cpd/rrb-guidance-oncontinuing-professional-development.pdf [Accessed online $27^{\text {th }}$ Apr 2021].

CRAFT JA, HUDSON PB, PLENDERLEITH MB, GORDON CJ (2017) Registered nurses' reflections on bioscience courses during the undergraduate nursing programme: an exploratory study. J Clin Nurs, 26(11-12): 1669-1680.

DAVIS D, O'BRIEN MAT, FREEMANTLE N, WOLF FM, MAZMANIAN P, TAYLOR-VAISEY A (1999) Impact of formal continuing medical education: do conferences, rounds, and other traditional continuing education activities change physician behaviour or health care outcomes? JAMA, 282: 867-874.

DE BOO DW, MARSHALL E, ERSKINE B, KOUKOUNARAS J, KAVNOUDIAS H, THOMSON KR (2020) Evaluation of a radiographer-led peripherally inserted central catheter insertion service. J Med Imaging Radiat Oncol, 64(4): 471-476.

DRAKE RL, MCBRIDE JM, LACHMAN N, PAWLINA W (2009) Medical education in the anatomical sciences: The winds of change continue to blow. Anat Sci Ed, 2(6): 253-259.

DOUGHTY J, HODGSON D (2009) Evaluation of a new clinical support model in radiotherapy practice. Nurse Educ Pract, 9(1): 28-35.

GORE JC (2020) Artificial intelligence in medical imaging. Magn Reson Imaging, 68: A1-A4.

GREHAN J, BUTLER ML, LAST J, RAINFORD L (2018) The introduction of mandatory CPD for newly state registered diagnostic radiographers: An Irish perspective. Radiography, 24(2): 115-121.

HALL AS, DURWARD BR (2009) Retention of anatomy knowledge by student radiographers. Radiography, 15(3): e22-e28.

HARDY M, SNAITH B (2009) Radiographer interpretation of trauma radiographs: Issues for radiography education providers. Radiography, 15(2): 101-105.

HENWOOD S, EDIE J, FLINTON D, SIMPSON R (1998) Continuing professional development - a re-examination of the facts. Radiography, 4(1): 5-8.

HEPTONSTALL NB, ALI T, MANKAD K (2016) Integrating radiology and anatomy teaching in medical education in the UK-the evidence, current trends, and future scope. Acad Radiol, 23(4): 521-526.

MARSHALL G, PUNYS V, SYKES A (2008) The continuous professional development (CPD) requirements of radiographers in Europe: An initial survey. Radiography, 14(4): 332-342.

MARTIN K, BESSELL NJ, SCHOLTEN I (2014) The perceived importance of anatomy and neuroanatomy in the practice of speechLanguage pathology. Anat Sci Educ, 7(1): 28-37.

MCLACHLAN JC, PATTEN D (2006) Anatomy teaching: ghosts of the past, present and future. Med Educ, 40(3): 243-253.

MINOGUE S, GILLHAM C, KEARNEY M, MULLANEY L (2019) Intravenous contrast media in radiation therapy planning computed tomography scans - Current practice in Ireland. Tech Innov Patient Support Radiat Oncol, 1(12): 3-15.

PATEL KM, MOXHAM BJ (2006) Attitudes of professional anatomists to curricular change. Clin Anat, 19(2): 132-141.

SHANAHAN M (2016) Student perspective on using a virtual radiography simulation. Radiography, 22(3): 217-222.

STERLING M, LEUNG P, WRIGHT D, BISHOP TF (2017) The use of social media in Graduate Medical Education: A systematic review. Acad Med, 92(7): 1043-1056.

SUGAND K, ABRAHAMS P, KHURANA A (2010) The anatomy of anatomy: A review for its modernization. Anat Sci Educ, 3(2): 83-93.

TURNEY BW (2007) Anatomy in a Modern Medical Curriculum. Ann R Coll Surg Engl, 89(2): 104-107.

WALLACE S, MAY SA (2016) Assessing and enhancing quality through outcomes-based continuing professional development (CPD): a review of current practice. Veterinary Rec, 179(20): 515-520.

WALSH R, CRAIG A (2016) Radiation Therapists' and Diagnostic Radiographers' participation in continuing professional development and knowledge of regulatory body registration. J Radiother Pract, 15(2): 150-160.

WAREING A, BUISSINK C, HARPER D, OLESEN MG, SOTO M, BRAICO S, VAN LAER P, GREMION I, RAINFORD L (2017) Continuing professional development (CPD) in radiography: A collaborative European metaethnography literature review. Radiography, 23(Suppl.1): S58-S63.

WATERSTON S, STEWART I (2005) Survey of clinicians' attitudes to the anatomical teaching and knowledge of medical students. Clin Anat, 18(5): 380-384. 